\title{
International collaborations in research to be a mainstay of modern research-a huge impact at enhancing research and awareness in the developing world
}

\section{News}

Biological systems have a complex nature. Studies on biological systems or research have evolved to be multidisciplinary in nature. Modern technologies from diverse fields have converged to address scientific problems in biology. Collaborative research arose through vision of scientists who realise the complexity of biological systems. Today diverse research groups collaborate to understand these complex biological systems. This news section examines the merits of such collaborative research and emphasizes expansion of this concept. Already international collaborations are evident as indicated in footer section of this article. ${ }^{1,2}$ This short article hopes to highlight some of the advantages and potential of such collaborations. So far, a large proportion of collaborations are confined to science powerhouses in the developed world: United States, England, and other nations of the European Union, Japan, Korea and more recently India and China. ${ }^{3,4}$ International collaborative events enhance awareness to the latest development in Science \& technology. Technology transfer \& hands on experience of such technologies enhances overall scientific experience. Exchange of personnel and interaction sessions amongst diverse group of scientist's also likely favours' tolerance for cultural differences among science peers. ${ }^{5}$ I believe that we are at present poised to undergo a remarkable transformation and enrichment of collaborative activities. Expansion of internet based technologies and telecommunications is likely to bridge this gap further. At the forefront, a country in Africa, Ethiopia has recently pledged a US \$40million investment to enhance its internet services. ${ }^{6}$ Due to expanding mobile phone coverage, about 6billion people have access to mobile phones ( $>$ than $75 \%$ of World population). Hence internet and mobile based internet has increased the number of people connected to internet-with global connectivity (Source GSMA Mobile Economy).

Although advancement of science and technology may be easily understood from such collaborations the benefits in terms of sociological and cultural aspects are probably less apparent. So far, the developing world lags behind in collaborative efforts. This needs to change in favour of developing countries particularly in Africa, South America and South East Asia. The author has experience of teaching both in the developed and developing nations (USA, India and Currently in Ethiopia). My interaction with scientific personnel and students has allowed me to appreciate the benefit for such collaborations. The results of a small peer group study aimed at examining the impact of scientific interactions on a global scale on different parameters is presented in Table 1 Below.

Briefly a survey using volunteers at a private University in North India was conducted: Volunteers were asked to assess Four Collaboration advantages
Volume 2 Issue 5 - 2015

\author{
Sonal Malhotra \\ Department of Biotechnology, Addis Ababa Science and \\ Technology University, Ethiopia \\ Correspondence: Sonal Malhotra, Associate Professor \\ Department of Biotechnology, Addis Ababa Science and \\ Technology University, Addis Ababa, Ethiopia, \\ Email sonalmalhotra07@gmail.com
}

Received: December 31, 2014 | Published: October 12, 2015

i. Technology transfer \& Hands on experience,

ii. Exchange of personnel \& training

iii. Awareness of Technology impact \&

iv. Bridging the linguistic and Cultural Gap for their individual merit. Among a volunteer pool of 50, respondents voted and the data was pooled and an impact factor was calculated.

Impact factor indicates the percentage of volunteers that voted for a particular advantage as the most important aspect of cross border or international collaboration. For instance an impact factor of 0.3 indicates that about $30 \%$ of the volunteer voted for that collaboration advantage as the most significant advantage for collaboration to occur. Looking then at the data represented in Table 1 below, it appears that most volunteers considered $1 \& 2$ as equally important with 4 being the least significant. In addition it appears from this pilot study that volunteers representing students from a Biology class were aware of international collaborative efforts and its potential.

*Impact factor was determined by conducting a survey in a private Indian University for the importance or impact of Advantage listed above. The impact factor represents the rounded off percentage from about 50 respondents to the survey. In Table 1 above we list a small number of advantages and their impact determined by a survey based method. There was less awareness of Culture and Linguistic issues facing personnel engaged in collaborative efforts. Developing countries lacking infrastructure and laboratory resources stand to gain a lot in these areas. Interestingly most developing regions exhibit tremendous biodiversity (Africa, South America and Central and South East Asia in particular). Many unique and medicinally important plants have been identified here and their biological characterization can benefit from collaborations between the developed countries (Europe Americas and Asia) and the developing world (Africa and South East Asia). A study by Victor Kuete and Thomas Efferth 
exemplifies this. They identified biomedicinal plants through an extensive internet based search and then identified compounds in these plants that exhibit antitumor activity. This is a study between University of Cameroon in Africa and University of Mainz, Germany. ${ }^{7}$ Such Collaborations can provide resource optimization: where developing nations can tap into resources located in developed nations besides obtaining financial aid and provide the world with new medicinal products. Another example of biological important antimicrobial agent is Persea Lingue (Found in Chile South America) derived bioactive agent kaempherol rhamnoside. This compound can have an inhibitory effect on Staphylococcus efflux pump NorA. ${ }^{8}$ In conclusion it seems collaborative efforts need to be extended \& enhanced further in developing nations to raise awareness and skill set training and overall science upliftment.

Table I Research/teaching collaborations: advantage \& impact

\begin{tabular}{ll}
\hline Collaboration advantage & Impact factor* \\
\hline Technology Transfer and Hands on experience & 0.3 \\
Exchange of Personnel and training & 0.3 \\
Increased awareness to modern technology & 0.25 \\
Bridging the culture and linguistic gap & 0.15 \\
\hline
\end{tabular}

\section{Acknowledgements}

None.

\section{Conflict of interest}

Author declares that there is no conflict of interest.

\section{References}

1. Boston University International Office.

2. IIT Bhuwaneshwar MOU with University of Edinburgh, UK and University of Western Ontario, Canada.

3. International Collaboration between National Human Genome Research Institute and East European Nations.

4. International Health division ICMR

5. Spencer Rodgers J, Timothy McGovern. Attitudes towards the Culturally Different: The role of intercultural Communication barriers, affective responses, consensual stereotypes and perceived threat. International Journal of Intercultural relations. 2002;26(6):609-631.

6. http://news.bbc.co.uk/2/hi/technology/4416073.stm

7. Victor Kuette, Thomas Efferth. African Flora has the potential to fight multidrug resistance of Cancer. BioMed Research International. 2015:24.

8. Holler JG, Christensen SB, Slotved HC, et al. Novel inhibitory activity of staphylococcus aureus efflux pump NorA, by Kaempherol rhamnoside extracted from Persea lingue Nees. J Antimicrob Chemother. 2012;67(5):1138-1144. 